\title{
Volatility in the Italian Stock Market: an Empirical Study
}

\author{
Marco Raberto ${ }^{\mathrm{a}, \mathrm{c}}$, Enrico Scalas ${ }^{\mathrm{a}, \mathrm{d}}$, Gianaurelio Cuniberti a,b,1, \\ and Massimo Riani ${ }^{\mathrm{a}, \mathrm{c}}$. \\ ${ }^{a}$ Istituto Nazionale per la Fisica della Materia, Unità di Genova \\ ${ }^{\mathrm{b}}$ Max-Planck-Institut für Physik komplexer Systeme, \\ Nöthnitzer Straße 38, D-01187 Dresden, Germany \\ ${ }^{\mathrm{c}}$ Dipartimento di Fisica, Università di Genova, via Dodecaneso 33, I-16142 \\ Genova, Italy \\ ${ }^{\mathrm{d} D i p a r t i m e n t o ~ d i ~ S c i e n z e ~ e ~ T e c n o l o g i e ~ A v a n z a t e, ~ U n i v e r s i t a ̀ ~ d e l ~ P i e m o n t e ~}$ \\ Orientale, via Cavour 84, I-15100 Alessandria, Italy
}

\begin{abstract}
We study the volatility of the MIB30-stock-index high-frequency data from November 28, 1994 through September 15, 1995. Our aim is to empirically characterize the volatility random walk in the framework of continuous-time finance. To this end, we compute the index volatility by means of the log-return standard deviation. We choose an hourly time window in order to investigate intraday properties of volatility. A periodic component is found for the hourly time window, in agreement with previous observations. Fluctuations are studied by means of detrended fluctuation analysis, and we detect long-range correlations. Volatility values are $\log$-stable distributed. We discuss the implications of these results for stochastic volatility modelling.
\end{abstract}

Key words: Stochastic processes; random walk; statistical finance; econophysics PACS: 02.50. Ey, 02.50.Wp, 89.90.+n

Corresponding author: Massimo Riani (riani@ge.infm.it), url: www.ge.infm.it/econophysics

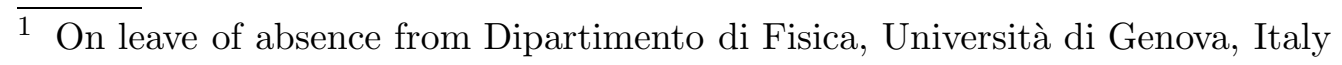

Preprint submitted to Elsevier Preprint

14 March 1998 


\section{Introduction}

In this paper, we study the stochastic properties of MIB30-stock-index volatility. The Black-Scholes/Merton (BS/M) framework for option pricing is based on the assumption of constant volatility [1]. In practice, volatility is time dependent and the characterization of its temporal evolution is one of the main task of the increasing number of physicists working in the financial field [2]. This problem has been thoroughly studied both by economists [3] and mathematicians [4]. However, the emphasis has been mainly given to analytically tractable problems.

Here, we use different approaches in the attempt to empirically characterize the MIB30-stock-index volatility. Among the various possible methods, we focus on the stochastic continuous-time volatility approach to option pricing.

The paper is divided as follows: in section 2, we present an outline of the stochastic-volatility theory; in section 3, the main empirical results are discussed; finally, conclusions are drawn in section 4 .

\section{Theory}

In continuous-time finance, stochastic volatility, $\sigma_{t}$, can be modeled by means of two stochastic differential equations [4], a two factor model:

$$
\begin{gathered}
d P(t)=\mu(P, t) P(t) d t+\sigma_{t} P(t) d w_{1}(t) \\
d \sigma_{t}=\alpha\left(\sigma_{t}, t\right) d t+\beta\left(\sigma_{t}, t\right) d w_{2}(t)
\end{gathered}
$$

where $\mu(P, t), \alpha\left(\sigma_{t}, t\right)$ and $\beta\left(\sigma_{t}, t\right)$ are deterministic functions of the spot price, $P(t)$, or of the stochastic volatility, $\sigma_{t}$, and of time, $t$; the volatility, $\sigma_{t}$, is a stochastic variable, $w_{1}$ and $w_{2}$ are standard one-dimensional Brownian motions with correlation $d\left\langle w_{1} w_{2}\right\rangle=\rho d t$ for some constant $\rho$. The two processes are independent if and only if $\rho=0$ [4]. The problem is then to find a unique solution $(\tilde{P}, \tilde{\sigma})$ for the system of stochastic differential equations (1) and (2). In the original $\mathrm{BS} / \mathrm{M}$ model, $\alpha$ and $\beta$ vanish and $\mu$ and $\sigma$ are constant.

If volatility is a stochastic process, continuous riskless hedging in the sense of BS/M (using an option and the underlying asset) is not possible [5]. This claim is based on a theorem concerning multi-factor stochastic models. In the particular case of the two-factor model of eqs. (1) and (2), in order to form a continuous riskless hedge, a financial instrument with price fully correlated 
to volatility would be necessary [5]. A clear and exhaustive introduction to multi-factor models can be found in Marco Avellaneda's tutorials [6].

If the ansatz of eq. (2) is accurate, the empirical analysis of stochastic volatility should lead to the determination of the coefficients $\alpha\left(\sigma_{t}, t\right)$ and $\beta\left(\sigma_{t}, t\right)[7]$, thus completely specifying its stochastic dynamics. In practice, this task can be very difficult, due to data incompleteness and to possible intrinsic mathematical difficulties. For instance, more than one set of the coefficients could well reproduce the known statistical properties of the volatility time series.

\section{Empirical Study}

We have analyzed MIB30 high frequency data from 28 November 1994 up to 15 September 1995. MIB30 is an official index of the Italian Stock Exchange, it is composed by the 30 Italian shares with the highest capitalization and trading volumes, and is recorded every minute. The data set is composed by over 80,000 data: 420 data for every trading day. Considering the series of index values, $P_{j \tau}$, where $\tau=1 \mathrm{~min}$ and $j=0, \ldots, 83579$, we divide our data into non overlapping intervals or time windows of length $T$. We choose a time horizon or time scale, $\Delta t$, which is an integer multiple of $\tau$. We compute the logarithmic returns related to every interval $T$ as follows:

$$
r_{n \Delta t}=\log \frac{P_{(n+1) \Delta t}}{P_{n \Delta t}}, n=0, \ldots, N-1
$$

where $N$ is such that $T=N \Delta t$.

Financial practitioners define historical volatility as the standard deviation of the logarithmic returns [8]. Following them, we estimate the volatility for every time window as follows:

$$
\bar{\sigma}=\sqrt{\frac{1}{N-1} \sum_{n=0}^{N-1}\left[r_{n \Delta t}-\bar{r}\right]^{2}}
$$

where $\bar{r}$ is the mean value given by:

$$
\bar{r}=\frac{1}{N} \sum_{n=0}^{N-1} r_{n \Delta t}
$$

If $\Delta t$ is measured as a fraction of year, we can define the annualized volatility:

$$
\bar{\sigma}_{\mathrm{an}}=\sqrt{\frac{1}{\Delta t}} \bar{\sigma}
$$


In order to investigate intraday properties of volatility, we chose a minutely time horizon and an hourly time window. The results of the 1393 annualized volatility estimates are plotted in Fig. 1.

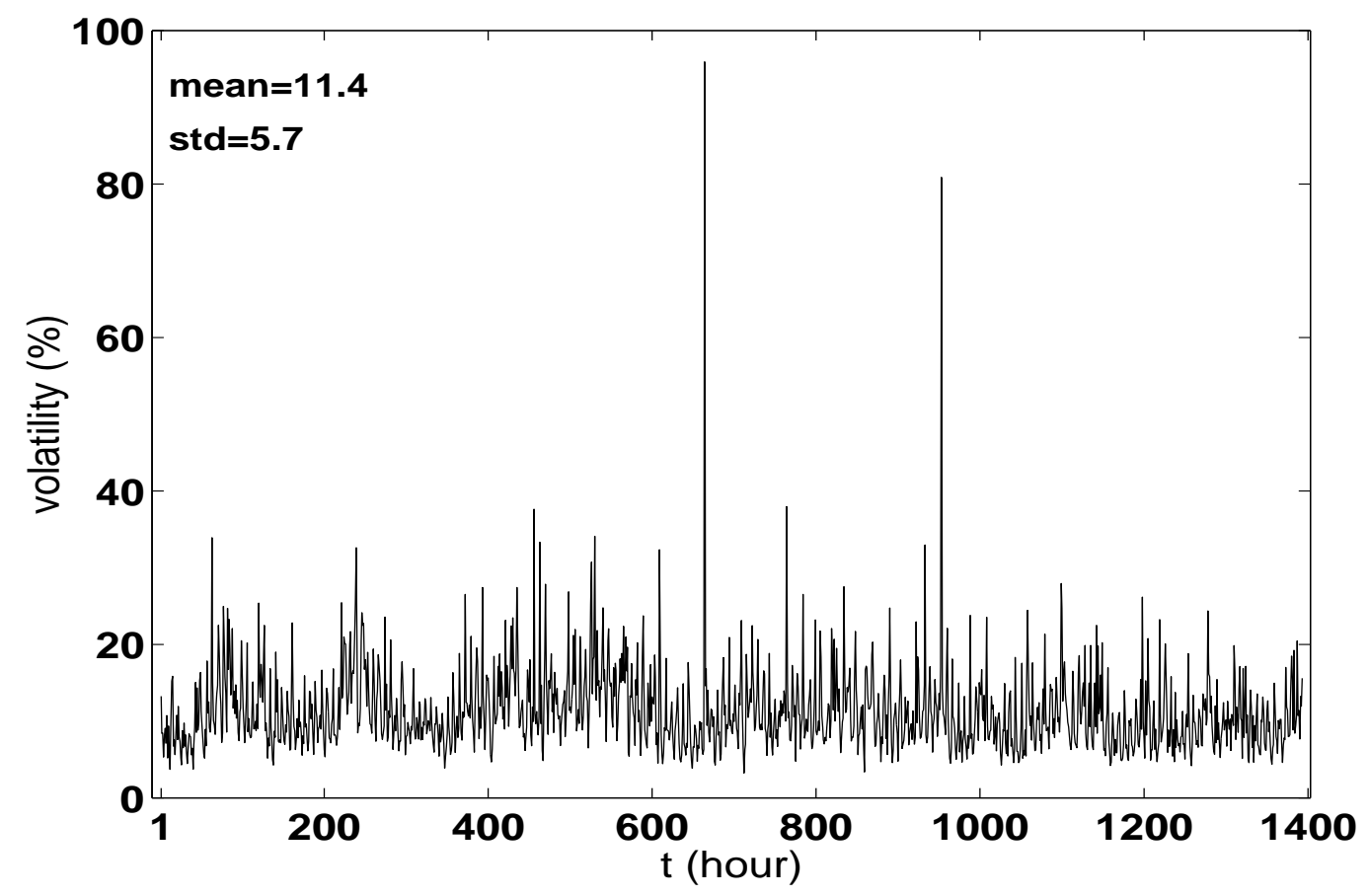

Fig. 1. Annualized volatility estimate; the time window is $1 h$; the time horizon is 1 min.

In Fig. 2 we present the power-spectrum-density estimate computed by means of the correlogram method [9]. The peak at $f / f_{s}=1 / 7$ is due to a daily periodicity of the volatility values. In fact, in a day there are seven trading hours and with an hourly time window we get seven volatility estimates per day. Indeed, intra-day volatility is U-shaped: it is higher at the opening and at the closure of the market. This fact has already been observed by economists, and it is also known in the physics literature [10]; it probably reflects the lower trading activity around noon. As a further remark, we are not able to detect any low frequency seasonality or observe a clear flicker behaviour at low frequencies [11], as our time series is less than one year long.

In Fig. 3, an estimate of the volatility probability density function is given. We compare the experimental histogram with a log-stable distribution whose parameters, $\delta$ and $\gamma$ are obtained from empirical data. Stable or Pareto-Lévy distributions [12] have been introduced in the sixties in finance and economics [13] and their scaling properties have been recently investigated in relation to the S\&P500 stock index[14]. Zero mean stable distributions are described by 


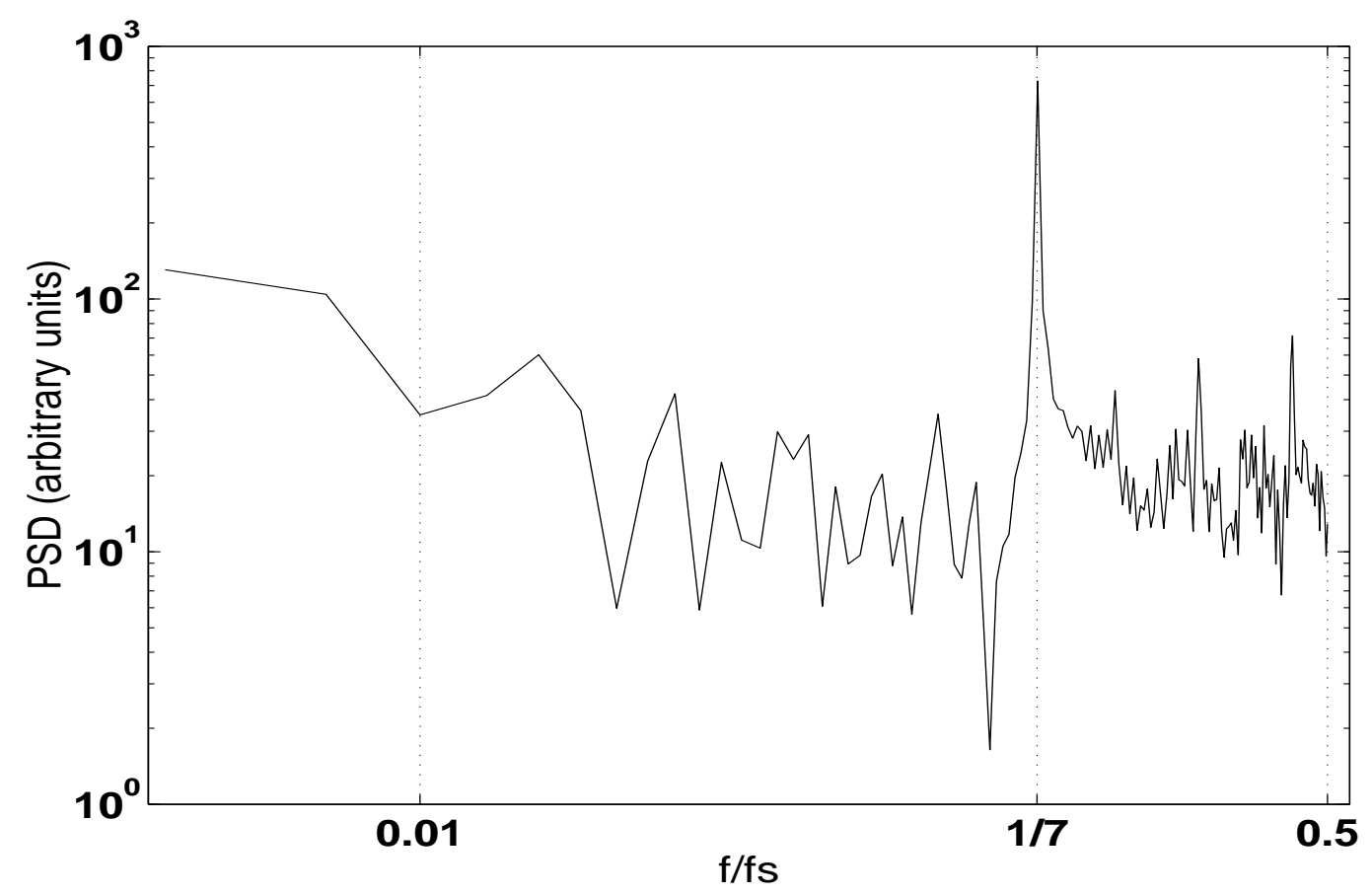

Fig. 2. Power spectrum density estimate. The sampling frequency, $f_{s}$, is equal to $1 /$ hour.

the following equation:

$$
P_{\delta, \gamma}(x)=\frac{1}{\pi} \int_{0}^{\infty} \exp \left(-\gamma q^{\delta}\right) \cos (q x) d q
$$

where $\delta=1$ and $\delta=2$ give, respectively, the well known Cauchy and Gauss stable distributions. A random variable is said to be log-stable distributed if its logarithm follows a stable distribution.

In order to determine the experimental points, the volatility range has been divided into fifty equal intervals (bins). From Fig. 1, it can be seen that there are two outliers. If the outliers are taken into account, a log-Lévy distribution with exponents $\delta=1.6$ and $\gamma=0.13$ gives an acceptable fit of the experimental data $\left(\chi^{2} \simeq 75\right.$ with 47 degrees of freedom), whereas the log-normal fit with mean value and standard deviation drawn from data does not agree in the tail region $\left(\chi^{2} \sim 10^{4}\right)$. Conversely, if the two outliers are rejected as bad data points, and a new histogram is computed using twenty bins, the log-normal fit is as good as the log-Lévy fit (for the $\log$-Lévy fit, $\chi^{2}$ is 29.2 , whereas for the $\log$-normal, it is 28.7 ; this time there are 17 degrees of freedom).

In Fig. 4, the results of detrended fluctuation analysis (DFA) are presented. DFA is used to investigate the presence of correlations in time series [10], [11], [15], [16]. A volatility walk is defined by means of the displacement $y(t)$ : 


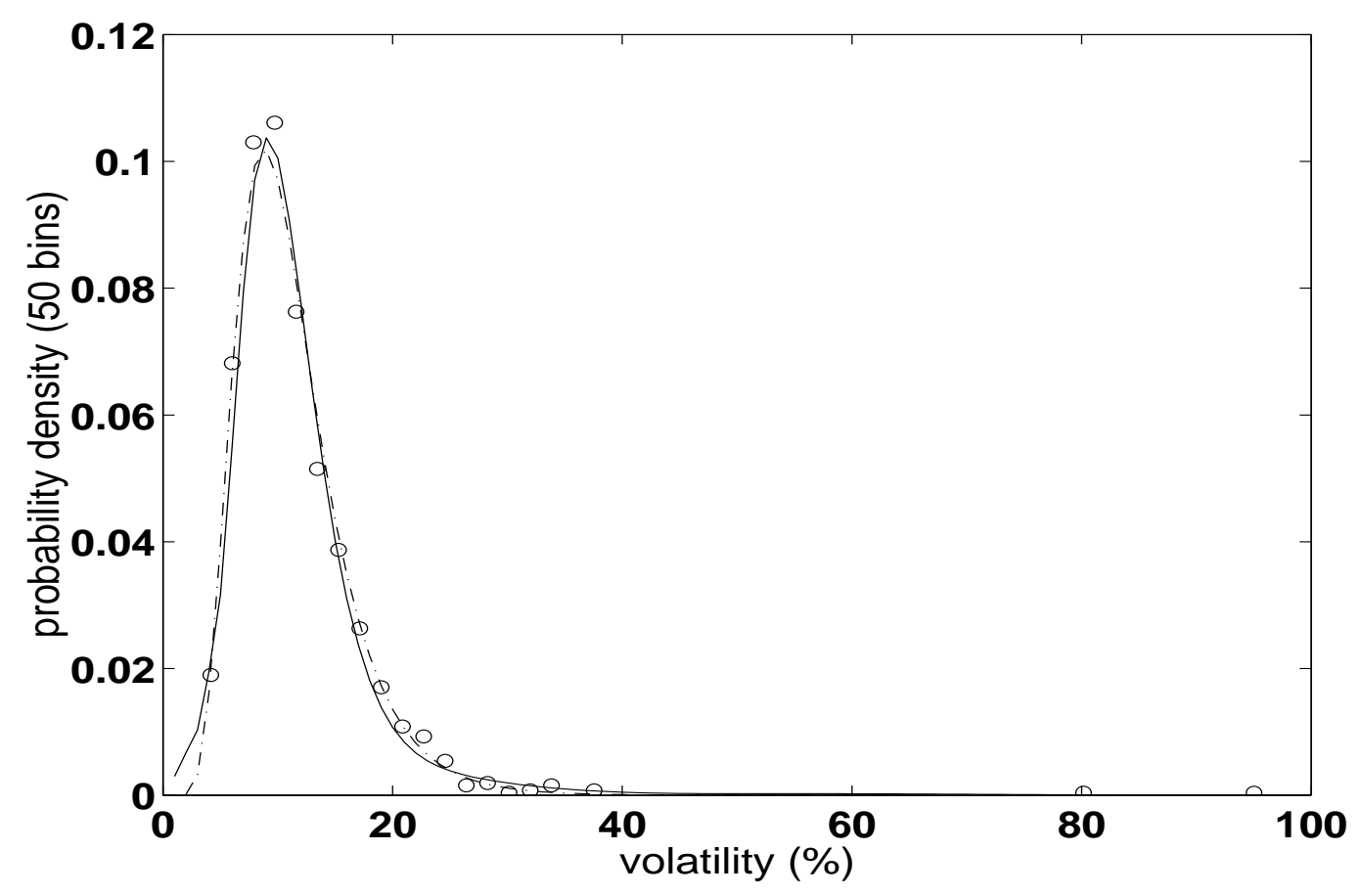

Fig. 3. Probability density function estimate; open circles: experimental histogram; solid line: $\log$-Lévy fit with $\delta=1.6$ and $\gamma=0.13$; dash-dotted line: log-normal fit with mean value $=2.34$ and standard deviation $=0.41$.

$$
y(t)=\sum_{i=1}^{t} \bar{\sigma}(i) .
$$

The mean square fluctuation, $F(t)$, around the average displacement is given by:

$$
F(t)=\sqrt{\left\langle\Delta y(t)^{2}\right\rangle-\langle\Delta y(t)\rangle^{2}}
$$

where $\Delta y(t)=y\left(t_{0}+t\right)-y\left(t_{0}\right)$, and $\langle\cdot\rangle$ is the average over all the initial steps $t_{0}$.

$F(t)$ follows the scaling law:

$$
F(t) \propto t^{z}
$$

If long-range correlations are present [17], the scaling exponent attains values $z \neq 1 / 2$.

The points in Fig. 4, top, have been computed according to Eq. (9) with overlapping subseries. Forty $F(t)$ estimates are plotted with their error bars. Errors are computed assuming a Gaussian distribution. The solid line is a least-square linear fit of the first six points, corresponding to one trading day; its slope gives $z=0.64 \pm 0.23$. The dash-dotted line is a least-square fit of the next thirty-four points and has a slope $z=0.76 \pm 0.16$. Therefore, from 

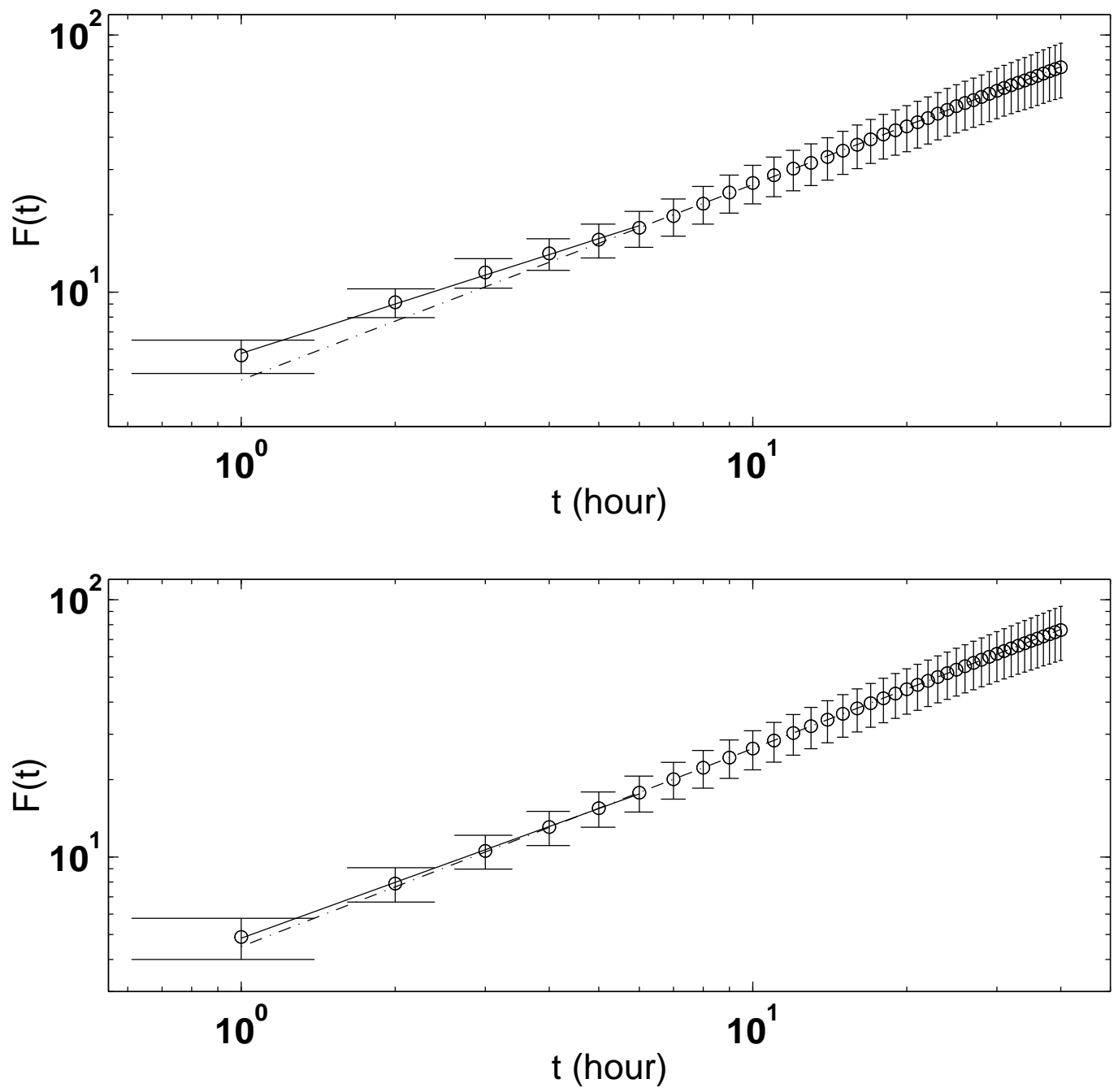

Fig. 4. DFA; top: $F(t)$ for the volatility walk; bottom: as top, but for de-U-shaped volatility walk, see text for explanation.

our data we cannot safely conclude that there is an exponent cross-over at $t=1$ trading day, as was found in ref. [11]. However, it is possible to argue that $z>1 / 2$; indeed, a linear fit of the forty points gives $z=0.72 \pm 0.10$.

In Fig. 4, bottom, we present $F(t)$ computed by "de-U-shaping" volatility values according to the following recipe:

$$
\sigma^{*}(i)=\bar{\sigma}(i) / n(i) \quad i=1, \ldots, 1393
$$

where the normalization coefficient, $n(i)$, is given by:

$$
n(i)=\frac{1}{q} \frac{\sum_{k=0}^{q-1} \bar{\sigma}(i+7 k)}{\langle\bar{\sigma}\rangle}, i=1, \ldots, 7
$$


where $q=1393 / 7$ and $n(i)$ has period 7 :

$$
n(i+7 h)=n(i) \quad h \in \mathbb{Z}
$$

In this way, the daily periodicity is removed and the intra-day estimate of $z$ is $0.72 \pm 0.26$, whereas the extra-day estimate is $z=0.77 \pm 0.16$. The possible cross-over seems to be suppressed, and the full linear least-square fit gives $z=0.76 \pm 0.10$, that is $z>1 / 2$.

\section{Summary and Conclusions}

In this paper, we have empirically studied the intra-day statistical properties of stochastic volatility for the MIB30 index. Stochastic volatility poses serious problems in contingent claim analysis and risk management. In many cases, risk managers consider a daily time window for computing volatility and perform daily hedging. However, the preliminary analysis of a recent liquidity crisis of a hedge fund seems to suggest that frequent intra-daily hedging could be necessary [18].

Empirical analysis constrains the form of the stochastic differential equations describing the time evolution of volatility.

In particular, we find in the MIB30 index that the volatility has a periodic behaviour with a one trading day period. Due to the limited amount of available data, we are not able to detect any low-frequency seasonality.

Probability-density-function estimates indicate that volatility is log-stable distributed; if outliers are taken into account a log-Lévy distribution gives a better fit than the usually assumed log-normal distribution [7].

Finally, the DFA results are compatible with the presence of long-range volatility correlations, but we cannot safely conclude that there is a crossover between intra-day and extra-day scaling exponents.

\section{Acknowledgements}

The authors are indebted to Rosario N. Mantegna for providing MIB30 data, for suggesting the work on stochastic volatility, and for helpful discussions. 


\section{References}

[1] R.C. Merton, Continuous Time Finance, Blackwell, Cambridge, MA (1990).

[2] G. Stix, Sci. Am., May 1998, 70

[3] J. Hull and A. White, Journal of Finance 42 (1987) 281; C. Ball and A. Roma, Journal of Financial and Quantitative Analysis 29 (1994) 589.

[4] M. Musiela and M. Rutkowski, Martingale Methods in Financial Modelling, Springer, Berlin, (1997), pages 154-158 and references therein.

[5] Y. Campbell, A.W. Lo and A. C. MacKinlay, The Econometrics of Financial Markets, Princeton University Press, Princeton, New Jersey (1997), page 380 and references therein.

[6] See: www.math.nyu.edu/faculty/avellane/risk.html

[7] P. Wilmott and A. Oztukel, International Journal of Theoretical and Applied Finance 1 (1998) 175.

[8] N.A. Chriss, Black-Scholes and Beyond, IRWIM, Chicago (1997).

[9] S.L. Marple Jr, Digital Spectral Analysis with Applications, Prentice-Hall, Englewood Cliffs, New York, 1987.

[10] P. Cizeau, Y. Liu, M Meyer, C.-K. Peng, and H.E. Stanley, Physica A 245 (1997) 441.

[11] Y. Liu, P. Cizeau, P. Gopikrishnan, M. Meyer, C.-K. Peng, and H.E. Stanley, Volatility Studies of the SESP 500 Index, in J. Kertesz and I. Kondor eds., Econophysics, an Emerging Science, Kluwer, Dordrecht, 1998.

[12] P. Lévy, Théorie de l'Addition des Variables Aléatoires, Gauthier-Villars, Paris (1937).

[13] B. Mandelbrot, Journal of Business 36 (1963) 394.

[14] R.N. Mantegna and H.E. Stanley, Nature 376 (1995) 46.

[15] E. Scalas, Physica A 253 (1998) 394.

[16] N. Vandewalle and M. Ausloos, Physica A 246 (1997) 454.

[17] E.W. Montroll, J.L. Lebowitz (Eds.), Studies in Statistical Mechanics, vol. XI, North-Holland, Amsterdam, 1984, pp. 1-122.

[18] A New Approach to Financial Risk, The Economist 349, October 17th 1998, p. 15. 